\title{
A Short Survey on Fault Diagnosis in Wireless Sensor Networks
}

\author{
Zeyu Zhang ${ }^{1,2}$, Lei Shu ${ }^{1,2}$, Amjad Mehmood ${ }^{1}$, Li Yan $^{1}$, and Yu Zhang ${ }^{2}$ \\ 1 Guangdong Provincial Key Lab of Petrochemical Equipment Fault Diagnosis, \\ Guangdong University of Petrochemical Technology, China, 525000 \\ 2 School of Engineering, University of Lincoln, UK \\ \{lei-shu, zeyu-zhang, dramjad.mehmmood, li.yan0823\}@outlook.com \\ YZhang@lincoln.ac.uk
}

\begin{abstract}
Fault diagnosis is one of the most important and demandable issues of the network. It makes the networks reliable and robust to operate in the normal way to handle almost all types of faults or failures. Additionally, it helps sensor nodes to work smoothly and efficiently till the end of their lifetime. This short survey paper not only presents a clear picture of the recent proposed techniques, but also draws comparisons and contrasts among them to diagnose the potential faults. In addition, it proposes some potential future-work directions which would lead to open new research directions in the field of fault diagnosis.
\end{abstract}

Key words: Wireless Sensor Networks, Fault diagnosis, Reliability

\section{Introduction}

Wireless Sensor Networks(WSNs) consist of a large number of low-cost, spatially distributed, small-in size, limited computation, storage and communication power sensor nodes that are deployed across the monitoring area. These nodes perform sensing, processing, communication and coordination of information with each other to achieve the common objectives autonomously. Due to recent advancement in wireless communication and electronics, micro-electromechanical system (MEMS) enables the resource constrained multi-functional sensor nodes to untetheredly communicate with each other in short distances. These constraints on the network makes it different form the other existing wireless networks. In WSNs, each small sensor node is composed of the following main components such as: (a) processing (b) communication (c) computation (d) power source (e) external memory (f) one or more sensors.

\section{Fault diagnosis approach}

According to the architecture, fault diagnosis model in WSNs consists of the following three types of approaches to handle faults: (i) Model-based or Centralized. (ii) Model-less or Distributed (iii) Model-based distributed or Hybrid 
approaches, each one of which is explained in detail with the help of most recent relevant work available in the literature, see in Figure 1.

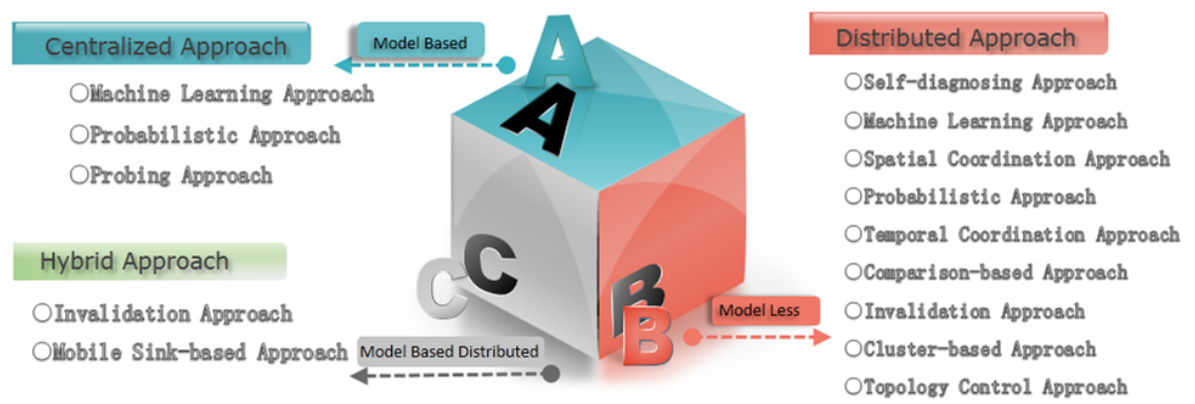

Fig. 1. Fault diagnosis categorization and approaches

\subsection{Model-based or Centralized approach}

As name indicates, an ultra-reliable centralized sensor node called sink node, with large storage, interrupted power supply and high computational power, is placed logically or geographically in the center. The base station or sink node periodically injects health requests or queries messages to determine the state of each sensor node deployed in the field. There are many techniques available in the literature of WSNs which have followed the centralized approaches for the purpose of fault detection and diagnosis.

\subsection{Model-less or Distributed approach}

Unlike model-based or centralized approach, each sensor node in model-less or distributed approach takes decision about their health status by gathering and analyzing diagnostic response results from the neighboring nodes. Then it updates the BS accordingly. Therefore, the model-less approaches transfer a little information to the BS that helps in prolonging the lifetime. It further reduces a lot of traffic overhead, and minimizes the end-to-end delay over the network. There are many recent techniques in the literature which have followed the distributed approaches for fault diagnosis and detection.

\subsection{Distributed model-based or Hybrid approach}

It combines the advantages of both centralized (model based) and distribute approach (model-less), and avoids the limitations of both. According to literature, the model based distributed approaches are preferred in terms of reliability, robustness, energy efficiency, and minimizes traffic overhead. So these approaches 
bridge the gap between the centralized, and distributed approaches. There are a few techniques available in the literature based on the subject cited above.

Table I presents some of the most important and recent protocols, which have been presented in the literature, are classified on the basis of different parameters of fault diagnosis. It would help the researchers to find the current trends of the protocols. It also illustrates the advantages and limitation of each. In order to contribute a more demanded-able protocol to be robust, reliable, energy-efficient for the domain.

\section{Open research Challenges}

WSNs have steadily become a cutting edge technology of the $21^{\text {st }}$ century for the development of wireless sensor applications. It is the most important area because its applications applying to almost all walks of life. Due to its importance, a lot of work has been performed in last one decade, nevertheless the area still demands more work to be done in order to fulfill the current requirements. The followings are some challenges listed below which need be focused:

1. More intelligent algorithms are required for the purpose of fault diagnosis and detection

2. Nodes are required to be diagnosed while performing their usual task simultaneously

3. The network must be prepared for load balancing efficiently specially in the case of multi-media sensor nodes

4. It is required to be adaptive to dynamics changes occurring such as topology, transmission ranges etc.

5. Intelligent movable robot needs to be proposed for diagnosis and detection

6. QoS-based fault diagnosis needs to be concentrated on network energy consumption and link quality.

7. Malicious activities and threats are required to be tracked in order to operate uninterruptedly.

8. Damaged link diagnosis and detection need to be addressed in large scale WSNs.

9. After diagnosing the faulty nodes, they are required to be recovered or reused as much as possible (communication, storage, computation)

10. A cross layer approach to deal with the reliability and robustness of the network

\section{Conclusion}

This short survey provides a big picture of promising techniques for fault diagnosis and detection existing till date. It also elaborates their strong and weak points. It is believed that this survey will be appreciated, helpful in proposing more robust, reliable, scalable,real-time, mobile, energy-efficient, and intelligent protocols in the near future. 
Table 1. Analysis with respect to different fault diagnosis parameters

\begin{tabular}{|c|c|c|c|c|c|c|c|c|c|c|c|c|}
\hline \multirow{2}{*}{ Author } & \multirow{2}{*}{ Year } & \multicolumn{3}{|c|}{ Diagnosis Network } & \multicolumn{3}{|c|}{ Diagnosis view } & \multicolumn{2}{|c|}{ Fault Persistence } & \multicolumn{2}{|c|}{ Fault Type } & \multirow[b]{2}{*}{ Approach } \\
\hline & & $\begin{array}{l}\text { Dis- } \\
\text { tribut- } \\
\text { ed }\end{array}$ & $\begin{array}{l}\text { Cen- } \\
\text { tral- } \\
\text { ized }\end{array}$ & Hybrid & Local & $\begin{array}{l}\text { Glob- } \\
\text { al }\end{array}$ & $\begin{array}{c}\text { Perma- } \\
\text { nent }\end{array}$ & $\begin{array}{l}\text { Inter- } \\
\text { mit- } \\
\text { tent }\end{array}$ & $\begin{array}{l}\text { Tran- } \\
\text { sient }\end{array}$ & Hard & Soft & \\
\hline $\begin{array}{l}\text { Shahram et } \\
\text { al. [3] }\end{array}$ & 2013 & $\checkmark$ & & & $\checkmark$ & $\checkmark$ & $\checkmark$ & & $\checkmark$ & $\checkmark$ & $\checkmark$ & $\begin{array}{l}\text { Self- } \\
\text { diagnosing }\end{array}$ \\
\hline $\begin{array}{l}\text { Miao et } \\
\text { al. [16] }\end{array}$ & 2013 & & $\checkmark$ & & & $\checkmark$ & & $\checkmark$ & $\checkmark$ & & $\checkmark$ & $\begin{array}{l}\text { Machine } \\
\text { Learning }\end{array}$ \\
\hline $\begin{array}{l}\text { Kulla et } \\
\text { al. [12] }\end{array}$ & 2013 & $\checkmark$ & & & $\checkmark$ & & & $\checkmark$ & $\checkmark$ & $\checkmark$ & $\checkmark$ & $\begin{array}{l}\text { Machine } \\
\text { Learning }\end{array}$ \\
\hline $\begin{array}{l}\text { Banerjee et } \\
\text { al. }[4]\end{array}$ & 2014 & & & $\checkmark$ & $\checkmark$ & $\checkmark$ & $\checkmark$ & $\checkmark$ & $\checkmark$ & $\checkmark$ & $\checkmark$ & $\begin{array}{l}\text { Spatial- } \\
\text { temporal } \\
\text { Coordina- } \\
\text { tion }\end{array}$ \\
\hline $\begin{array}{l}\text { Chanak et } \\
\text { al. [5] }\end{array}$ & 2013 & $\checkmark$ & & & $\checkmark$ & & $\checkmark$ & & $\checkmark$ & $\checkmark$ & $\checkmark$ & $\begin{array}{l}\text { Spatial } \\
\text { Coordina- } \\
\text { tion }\end{array}$ \\
\hline $\begin{array}{l}\text { Alessandra } \\
\text { et al. [7] }\end{array}$ & 2013 & $\checkmark$ & & & $\checkmark$ & & & $\checkmark$ & $\checkmark$ & & $\checkmark$ & $\begin{array}{l}\text { Proba- } \\
\text { bilistic }\end{array}$ \\
\hline $\begin{array}{l}\text { Dima et } \\
\text { al. [10] }\end{array}$ & 2013 & $\checkmark$ & & & $\checkmark$ & & $\checkmark$ & $\checkmark$ & $\checkmark$ & $\checkmark$ & $\checkmark$ & $\begin{array}{l}\text { Spatial } \\
\text { Coordina- } \\
\text { tion }\end{array}$ \\
\hline $\begin{array}{l}\text { Bill et } \\
\text { al. [13] }\end{array}$ & 2014 & & $\checkmark$ & & & $\checkmark$ & & $\checkmark$ & $\checkmark$ & & $\checkmark$ & $\begin{array}{l}\text { Proba- } \\
\text { bilistic }\end{array}$ \\
\hline $\begin{array}{l}\text { Arunanshu } \\
\text { et al. [15] }\end{array}$ & 2014 & $\checkmark$ & & & $\checkmark$ & & $\checkmark$ & $\checkmark$ & & $\checkmark$ & $\checkmark$ & $\begin{array}{l}\text { Comparisor } \\
\text { based }\end{array}$ \\
\hline $\begin{array}{l}\text { Manmath } \\
\text { et al. [21] }\end{array}$ & 2014 & $\checkmark$ & & & $\checkmark$ & & $\checkmark$ & $\checkmark$ & & $\checkmark$ & $\checkmark$ & $\begin{array}{l}\text { Spatial } \\
\text { Coordina- } \\
\text { tion }\end{array}$ \\
\hline $\begin{array}{l}\text { Mehdi et } \\
\text { al. [2] }\end{array}$ & 2014 & $\checkmark$ & & & $\checkmark$ & & $\checkmark$ & $\checkmark$ & $\checkmark$ & $\checkmark$ & $\checkmark$ & $\begin{array}{l}\text { Cluster- } \\
\text { based }\end{array}$ \\
\hline $\begin{array}{l}\text { Yu et } \\
\text { al. }[25]\end{array}$ & 2014 & & $\checkmark$ & & & $\checkmark$ & $\checkmark$ & $\checkmark$ & $\checkmark$ & $\checkmark$ & $\checkmark$ & $\begin{array}{l}\begin{array}{l}\text { Model- } \\
\text { based }\end{array} \\
\end{array}$ \\
\hline $\begin{array}{l}\text { Arunanshu } \\
\text { et al. [15] }\end{array}$ & 2013 & $\checkmark$ & & & $\checkmark$ & & $\checkmark$ & $\checkmark$ & $\checkmark$ & $\checkmark$ & $\checkmark$ & \begin{tabular}{|l} 
Invalida- \\
tion \\
\end{tabular} \\
\hline $\begin{array}{l}\text { M. Panda } \\
\text { et al. [18] }\end{array}$ & 2015 & $\checkmark$ & & & $\checkmark$ & & $\checkmark$ & $\checkmark$ & $\checkmark$ & $\checkmark$ & $\checkmark$ & $\begin{array}{l}\text { Self- } \\
\text { diagnosing }\end{array}$ \\
\hline $\begin{array}{l}\text { Yuan et } \\
\text { al. [26] }\end{array}$ & 2015 & $\checkmark$ & & & $\checkmark$ & & & $\checkmark$ & & & $\checkmark$ & $\begin{array}{l}\text { Proba- } \\
\text { bilistic } \\
\end{array}$ \\
\hline $\begin{array}{l}\text { Zafar et } \\
\text { al. }[27]\end{array}$ & 2015 & & & $\checkmark$ & $\checkmark$ & $\checkmark$ & & $\checkmark$ & $\checkmark$ & & $\checkmark$ & \begin{tabular}{|l|} 
Invalida- \\
tion \\
\end{tabular} \\
\hline $\begin{array}{l}\text { Dhal et } \\
\text { al. [8] }\end{array}$ & 2015 & & $\checkmark$ & & & $\checkmark$ & & $\checkmark$ & $\checkmark$ & & $\checkmark$ & $\begin{array}{l}\text { Topology } \\
\text { Control }\end{array}$ \\
\hline $\begin{array}{l}\text { Gong et } \\
\text { al. [9] }\end{array}$ & 2015 & & $\checkmark$ & & & $\checkmark$ & & $\checkmark$ & $\checkmark$ & & $\checkmark$ & Probing \\
\hline $\begin{array}{l}\text { Meenakshi } \\
\text { et al [19] }\end{array}$ & 2015 & $\checkmark$ & & & $\checkmark$ & & & $\checkmark$ & $\checkmark$ & & $\checkmark$ & $\begin{array}{l}\text { Majority } \\
\text { Voting } \\
\end{array}$ \\
\hline $\begin{array}{l}\text { Lo et } \\
\text { al. [14] }\end{array}$ & 2016 & & & $\checkmark$ & $\checkmark$ & $\checkmark$ & & $\checkmark$ & $\checkmark$ & & $\checkmark$ & $\begin{array}{l}\text { Spatial } \\
\text { Coordina- } \\
\text { tion }\end{array}$ \\
\hline $\begin{array}{l}\text { Chafig et } \\
\text { al. [23] }\end{array}$ & 2015 & $\checkmark$ & & & $\checkmark$ & $\checkmark$ & & $\checkmark$ & $\checkmark$ & & $\checkmark$ & $\begin{array}{l}\text { Proba- } \\
\text { bilistic }\end{array}$ \\
\hline $\begin{array}{l}\text { Jin et } \\
\text { al. [11] }\end{array}$ & 2015 & & $\checkmark$ & & $\checkmark$ & $\checkmark$ & & $\checkmark$ & $\checkmark$ & & $\checkmark$ & \begin{tabular}{|l}
$\begin{array}{l}\text { Model- } \\
\text { based }\end{array}$ \\
\end{tabular} \\
\hline $\begin{array}{l}\text { Mohammed } \\
\text { et al. [1] }\end{array}$ & 2015 & & & $\checkmark$ & & $\checkmark$ & $\checkmark$ & $\checkmark$ & $\checkmark$ & $\checkmark$ & $\checkmark$ & $\begin{array}{l}\text { Mobile } \\
\text { Sink-based }\end{array}$ \\
\hline $\begin{array}{l}\text { Christopher } \\
\text { et al. [17] }\end{array}$ & 2016 & & $\checkmark$ & & & $\checkmark$ & & $\checkmark$ & $\checkmark$ & & $\checkmark$ & $\begin{array}{l}\text { Topology } \\
\text { Control }\end{array}$ \\
\hline $\begin{array}{l}\text { Panigrahi } \\
\text { et al. [20] }\end{array}$ & 2016 & $\checkmark$ & & & $\checkmark$ & & & $\checkmark$ & $\checkmark$ & & $\checkmark$ & $\begin{array}{l}\text { Spatial } \\
\text { Coordina- } \\
\text { tion }\end{array}$ \\
\hline $\begin{array}{l}\text { Zhen et } \\
\text { al. [29] }\end{array}$ & 2016 & & $\checkmark$ & & & $\checkmark$ & & $\checkmark$ & $\checkmark$ & & $\checkmark$ & $\begin{array}{l}\text { Model- } \\
\text { based }\end{array}$ \\
\hline $\begin{array}{l}\text { Hongsheng } \\
\text { et al. [24] }\end{array}$ & 2016 & $\checkmark$ & & & $\checkmark$ & & $\checkmark$ & $\checkmark$ & $\checkmark$ & $\checkmark$ & $\checkmark$ & $\begin{array}{l}\text { Spatial } \\
\text { Coordina- } \\
\text { tion }\end{array}$ \\
\hline $\begin{array}{l}\text { Zhang et } \\
\text { al. [28] }\end{array}$ & 2016 & $\checkmark$ & & & $\checkmark$ & $\checkmark$ & $\checkmark$ & $\checkmark$ & $\checkmark$ & $\checkmark$ & $\checkmark$ & $\begin{array}{l}\text { Spatial } \\
\text { Coordina- } \\
\text { tion }\end{array}$ \\
\hline $\begin{array}{l}\text { Tang et } \\
\text { al. [22] }\end{array}$ & 2016 & $\checkmark$ & & & $\checkmark$ & $\checkmark$ & & $\checkmark$ & $\checkmark$ & & $\checkmark$ & $\begin{array}{l}\text { Machine } \\
\text { Learning }\end{array}$ \\
\hline $\begin{array}{l}\text { Chanak et } \\
\text { al. [6] }\end{array}$ & 2016 & & & $\checkmark$ & $\checkmark$ & $\checkmark$ & $\checkmark$ & $\checkmark$ & $\checkmark$ & $\checkmark$ & $\checkmark$ & $\begin{array}{l}\text { Mobile } \\
\text { Sink-based }\end{array}$ \\
\hline $\begin{array}{l}\text { Chanak et } \\
\text { al. [5] }\end{array}$ & 2016 & $\checkmark$ & & & $\checkmark$ & & $\checkmark$ & $\checkmark$ & & $\checkmark$ & $\checkmark$ & \begin{tabular}{|l|}
$\begin{array}{l}\text { Proba- } \\
\text { bilistic }\end{array}$ \\
\end{tabular} \\
\hline
\end{tabular}




\section{Acknowledgments}

This work is supported by International and Hong Kong, Macao \& Taiwan collaborative innovation platform and major international cooperation projects of colleges in Guangdong Province (No.2015KGJHZ026) and Guangdong University of Petrochemical Technology Internal Project 2012RC106. Lei Shu is the corresponding author.

\section{References}

1. Abo-Zahhad, M., Ahmed, S.M., Sabor, N., Sasaki, S.: Mobile sink-based adaptive immune energy-efficient clustering protocol for improving the lifetime and stability period of wireless sensor networks. IEEE Sensors Journal 15(8), 4576-4586 (2015)

2. Afsar, M.M.: Maximizing the reliability of clustered sensor networks by a faulttolerant service. In: Electrical and Computer Engineering (CCECE), 2014 IEEE 27th Canadian Conference on. pp. 1-8. IEEE (2014)

3. Babaie, S., Khosrohosseini, A., Khadem-Zadeh, A.: A new self-diagnosing approach based on petri nets and correlation graphs for fault management in wireless sensor networks. Journal of Systems Architecture 59(8), 582-600 (2013)

4. Banerjee, I., Chanak, P., Rahaman, H., Samanta, T.: Effective fault detection and routing scheme for wireless sensor networks. Computers \& Electrical Engineering 40(2), 291-306 (2014)

5. Chanak, P., Banerjee, I.: Fuzzy rule-based faulty node classification and management scheme for large scale wireless sensor networks. Expert Systems with Applications 45, 307-321 (2016)

6. Chanak, P., Banerjee, I., Sherratt, R.S.: Mobile sink based fault diagnosis scheme for wireless sensor networks. Journal of Systems and Software (2016)

7. De Paola, A., Lo Re, G., Milazzo, F., Ortolani, M.: Qos-aware fault detection in wireless sensor networks. International Journal of Distributed Sensor Networks $2013(2013)$

8. Dhal, R., Torres, J.A., Roy, S.: Detecting link failures in complex network processes using remote monitoring. Physica A: Statistical Mechanics and its Applications 437, 36-54 (2015)

9. Gong, W., Liu, K., Liu, Y.: Directional diagnosis for wireless sensor networks. IEEE Transactions on Parallel and Distributed Systems 26(5), 1290-1300 (2015)

10. Hamdan, D., Parissis, I., Hijazi, A., El Hassan, B., et al.: Test and diagnosis of wireless sensor networks applications. In: Computer and Information Technology (WCCIT), 2013 World Congress on. pp. 1-7. IEEE (2013)

11. Jin, X., Chow, T.W., Sun, Y., Shan, J., Lau, B.C.: Kuiper test and autoregressive model-based approach for wireless sensor network fault diagnosis. Wireless Networks 21(3), 829-839 (2015)

12. Kullaa, J.: Detection, identification, and quantification of sensor fault in a sensor network. Mechanical Systems and Signal Processing 40(1), 208-221 (2013)

13. Lau, B.C., Ma, E.W., Chow, T.W.: Probabilistic fault detector for wireless sensor network. Expert Systems with Applications 41(8), 3703-3711 (2014)

14. Lo, C., Lynch, J.P., Liu, M.: Distributed model-based nonlinear sensor fault diagnosis in wireless sensor networks. Mechanical Systems and Signal Processing 66, 470-484 (2016) 
15. Mahapatro, A., Khilar, P.M.: Fault diagnosis in wireless sensor networks: A survey. IEEE Communications Surveys \& Tutorials 15(4), 2000-2026 (2013)

16. Miao, X., Liu, K., He, Y., Papadias, D., Ma, Q., Liu, Y.: Agnostic diagnosis: Discovering silent failures in wireless sensor networks. IEEE Transactions on Wireless Communications 12(12), 6067-6075 (2013)

17. Oßner, C., Buchmann, E., Böhm, K.: Identifying defective nodes in wireless sensor networks. Distributed and Parallel Databases pp. 1-20 (2016)

18. Panda, M., Khilar, P.M.: Distributed self fault diagnosis algorithm for large scale wireless sensor networks using modified three sigma edit test. Ad Hoc Networks 25, 170-184 (2015)

19. Panda, M., Khilar, P.: Distributed byzantine fault detection technique in wireless sensor networks based on hypothesis testing. Computers \& Electrical Engineering 48, 270-285 (2015)

20. Panigrahi, T., Panda, M., Panda, G.: Fault tolerant distributed estimation in wireless sensor networks. Journal of Network and Computer Applications 69, 27-39 (2016)

21. Sahoo, M.N., Khilar, P.M.: Diagnosis of wireless sensor networks in presence of permanent and intermittent faults. Wireless Personal Communications 78(2), 15711591 (2014)

22. Tang, P., Chow, T.W.: Wireless sensor-networks conditions monitoring and fault diagnosis using neighborhood hidden conditional random field. IEEE Transactions on Industrial Informatics 12(3), 933-940 (2016)

23. Titouna, C., Aliouat, M., Gueroui, M.: Fds: fault detection scheme for wireless sensor networks. Wireless Personal Communications 86(2), 549-562 (2016)

24. Xu, H., Zhang, R., Lin, C., Ma, Y.: Novel approach of fault diagnosis in wireless sensor networks node based on rough set and neural network model. International Journal of Future Generation Communication and Networking 9(4), 1-16 (2016)

25. Yu, C.B., Hu, J.J., Li, R., Deng, S.H., Yang, R.M.: Node fault diagnosis in wsn based on rs and svm. In: Wireless Communication and Sensor Network (WCSN), 2014 International Conference on. pp. 153-156. IEEE (2014)

26. Yuan, H., Zhao, X., Yu, L.: A distributed bayesian algorithm for data fault detection in wireless sensor networks. In: 2015 International Conference on Information Networking (ICOIN). pp. 63-68. IEEE (2015)

27. Zafar, A., Wajid, B., Akram, B.A.: A hybrid fault diagnosis architecture for wireless sensor networks. In: International Conference on Open Source Systems \& Technologies (ICOSST). pp. 7-15. IEEE (2015)

28. Zhang, Y., Yuan, X.: Fault diagnosis in clustering wsn based on neighbor cooperation. In: 2016 Chinese Control and Decision Conference (CCDC). pp. 1803-1807. IEEE (2016)

29. Zhen, F., JingQi, F., Wei, S.: The gateway anomaly detection and diagnosis in wsns. In: Control and Decision Conference (CCDC), 2016 Chinese. pp. 2401-2406. IEEE (2016) 